\title{
Comparação de métodos na determinação de sensibilidade à vancomicina em Staphylococcus aureus resistente à meticilina
}

\author{
Comparison of methods to determination of vancomycin sensivity in methicillin-resistant Staphylococcus aureus
}

Caroline Manfredini'; Simone Ulrich Picoli; Ana Paula Becker ${ }^{3}$

\section{unitermos}

Testes de sensibilidade microbiana

Staphylococcus aureus resistente à meticilina

Vancomicina

\section{resumo}

Introdução: Infecções por Staphylococcus aureus resistentes à meticilina (MRSA) são cada vez mais comuns, tanto em ambiente hospitalar como na comunidade. O principal antimicrobiano utilizado no tratamento de infecções causadas por MRSA é a vancomicina e devido ao seu uso indiscriminado surgiram cepas com sensibilidade diminuída. A partir de 2009 foi estabelecido que o teste de sensibilidade à vancomicina deve ser feito por métodos de concentração inibitória mínima (MIC). Por isso, este trabalho teve por objetivo determinar a sensibilidade à vancomicina em cepas MRSA por meio de diferentes metodologias, comparando os valores de MIC obtidos. Metodologia: Foram analisadas 46 cepas de S. aureus resistentes à oxacilina provenientes do Hospital Mãe de Deus, de Porto Alegre, Rio Grande do Sul. As técnicas de MIC empregadas foram macrodiluição em caldo, método comercial Etest ${ }^{\circledR} \mathrm{e}$ automação Microscan Walk Away (Siemens ${ }^{\circledR}$ ). Resultados: Todas as cepas foram sensíveis à vancomicina mediante todas as metodologias, contudo o Etest ${ }^{\circledR}$ apresentou valores de MIC mais elevados quando em comparação com a macrodiluição. A automação teve resultados equivalentes aos demais métodos por apresentar o valor de sensível como $\leq 2 \mu \mathrm{g} / \mathrm{ml}$. Discussão e conclusão: $O$ método comercial Etest ${ }^{\circledR}$ superestima a MIC, porém é fácil e rápido de ser realizado; já a macrodiluição em caldo é uma metodologia muito trabalhosa e demorada. Os resultados do Microscan Walk Away 96 (Siemens ${ }^{\circledR}$ ) são precisos, mas não fornecem o valor exato da MIC, exigindo um teste adicional para avaliar a eficácia do tratamento. Em conclusão, o Etest ${ }^{\oplus}$ pode ser utilizado como uma alternativa na rotina laboratorial. abstract

Introduction: Methicillin resistant Staphylococcus aureus (MRSA) infections are increasing, both in hospitals and the community. Vancomycin is the main antibiotic used to treat MRSA infections and this widespread use led to the emergence of isolates with reduced susceptibility. Since 2009 it was established that the susceptibility test to vancomycin should be determined using minimum inhibitory concentration (MIC). Objective: Based on the above, this study aimed to determine vancomycin susceptibility in MRSA isolates comparing antimicrobial susceptibility methods recommended by Clinical and Laboratory Standards Institute (CLSI). Methodology: We analyzed 46 isolates of S. aureus resistant to methicillin from Mãe de Deus Hospital, in Porto Alegre, Brazil. MICs for $S$. aureus isolates were determined using Etest ${ }^{\circledR}$ strips and were correlated with broth macrodilution method, Etest ${ }^{\circledR}$ and MicroScan Walk Away 96 (Siemens $\left.{ }^{\circledR}\right)$. Results: All strains were susceptible to vancomycin by all methods, however, the Etest ${ }^{\circledR}$ strips showed higher MIC values compared with broth macrodilution. MicroScan Walk Away 96 (Siemens $^{\circledR}$ ) shows similar results compared with other methods for presenting the value of sensitive as $\leq 2 \mu \mathrm{g} / \mathrm{ml}$. Discussion and conclusion: The Etest ${ }^{\circledR}$ method overestimates the MIC values, however it is easily and quickly accomplished. In the other hand, broth macrodilution is very laborious and time consuming. MicroScan Walk Away $96\left(\right.$ Siemens $\left.^{\circledR}\right)$ results are accurate, but does not provide the exact MIC value requiring an additional test to estimate the effectiveness of treatment. In conclusion, the Etest ${ }^{\circledR}$ can be used as an alternative for routine monitoring. key words

Microbial sensitivity tests

Methicillin-resistant

Staphylococcus aureus

Vancomycin

\footnotetext{
1. Acadêmica de Biomedicina da Federação de Estabelecimento de Ensino Superior em Novo Hamburgo (FEEVALE).

2. Mestra em Microbiologia; professora adjunta da FEEVALE.

3. Biomédica do Laboratório do Hospital Mãe de Deus (Porto Alegre-RS).

0 trabalho foi realizado no Laboratório de Biomedicina da FEEVALE.
} 


\section{Introdução}

Infecções causadas por Staphylococcus aureus resistentes à meticilina (MRSA) estão se tornando cada vez mais comuns, acarretando $40 \%$ a $70 \%$ de todas as infecções estafilocócicas do mundo( ${ }^{(9)}$. Um estudo feito por Cuevas et al., na Espanha, demonstrou que a resistência a esse fármaco vem aumentando progressivamente, passando de 1,5\% em 1986 para 31,2\% em 2002. Além disso, o mesmo estudo descreveu o aumento das cepas encontradas na comunidade (CA-MRSA) ${ }^{(3)}$.

Diversos autores atribuem o surgimento de cepas MRSA de origem comunitária ao uso indiscriminado e empírico de antimicrobianos, promovendo a pressão seletiva dessa bactéria. $O$ aumento de casos de CA-MRSA pode ser considerado um grande problema de saúde pública e um desafio para os profissionais da saúde ${ }^{(16)}$.

Recentemente foi sugerido que as linhagens CAMRSA iriam substituir as hospitalares (HA-MRSA) devido à alta taxa de crescimento e à maior aptidão biológica ${ }^{(4)}$. Essa inversão gera grande preocupação, pois infecções sabidamente mais graves causadas por CA-MRSA acometerão pacientes mais debili$\operatorname{tados}^{(4)}$

O MRSA não sofre ação da maioria dos antibióticos betalactâmicos, excetuando o ceftobiprole, uma nova cefalosporina de amplo espectro(5), e o razupenem, um novo carbapenem ${ }^{(8)}$; ambos os fármacos apresentam ação antiMRSA. Dessa forma, habitualmente os fármacos de escolha para o tratamento de infecções causadas por essas bactérias pertencem à classe dos glicopeptídeos (vancomicina e teicoplanina), que agem impedindo a síntese da parede celular ${ }^{(17)}$. Mediante o amplo emprego de vancomicina na prática clínica, ocorreu o surgimento de $S$. aureus com sensibilidade reduzida a esse antimicrobiano e demais glicopeptídeos, denominados $S$. aureus com sensibilidade intermediária a vancomicina/glicopeptídeos (VISA/GISA) e S. aureus resistente a vancomicina/ glicopeptídeos (VRSA/GRSA)(25).

O perfil de sensibilidade de cepas MRSA diante da vancomicina deve ser determinado por meio da concentração inibitória mínima (MIC), uma vez que o teste de disco difusão não é confiável. O mesmo não diferencia $S$. aureus sensível daquele intermediário a esse fármaco(2).

\section{Objetivo}

Este estudo teve como objetivo determinar o perfil de sensibilidade à vancomicina em cepas MRSA por meio de diferentes metodologias, comparando os valores de MIC obtidos.

\section{Materiais e métodos}

\section{Amostras bacterianas}

Foram avaliadas 46 amostras de $S$. aureus previamente isoladas no laboratório de microbiologia do Hospital Mãe de Deus, Porto Alegre, Rio Grande do Sul. Elas apresentavam resistência à meticilina (MRSA) por automação (MicroScan Walk Away 96, Siemens ${ }^{\circledR}$, Deerfield, USA), confirmadas por métodos moleculares (gene mecA) e classificadas em CA-MRSA e HA-MRSA, segundo critérios epidemiológicos do Centers for Disease Control and Prevention (CDC) $)^{(1)}$. Os isolados preservados em caldo glicerinado a $-80^{\circ} \mathrm{C}$ foram repicados em ágar sangue de carneiro, incubados por 18 horas e enviados ao Laboratório de Biomedicina da Universidade Feevale, onde foram submetidos a novo repique (ágar sangue carneiro, $35^{\circ} \mathrm{C}, 20$ horas) e processados.

\section{Determinação da MIC}

A MIC foi determinada por meio de diluição em caldo (macrodiluição) e Etest ${ }^{\circledR}$ (AB Biodisk, Solna, Sweden).

A macrodiluição foi executada nas seguintes etapas:

- preparo de solução estoque de vancomicina $16 \mu \mathrm{g} /$ $\mathrm{ml}$ (Sigma-Aldrich Chemie, Steinheim, Germany) diluída desde 8 até $0,125 \mu \mathrm{g} / \mathrm{ml}(8 ; 4 ; 2 ; 1 ; 0,5$; 0,$25 ; 0,125 \mu \mathrm{g} / \mathrm{ml}$ ) em caldo Mueller Hinton (Himedia, Mumbai, India), pH 7,2 a 7,4;

- preparo de suspensão bacteriana equivalente a 0,5 na escala de McFarland em solução salina estéril a partir de cultura fresca de MRSA em ágar sangue de carneiro (20 horas);

- adição de $100 \mu \mathrm{l}$ da suspensão 0,5 McFarland em 9,9 ml de caldo Mueller Hinton (diluição 1:100) e distribuição de $500 \mu \mathrm{l}$ desta em cada um dos nove tubos da macrodiluição, exceto no controle de esterilidade. Um dos nove tubos continha somente $500 \mu \mathrm{l}$ de caldo Mueller Hinton (controle de inóculo). Todos os tubos foram incubados a $35^{\circ} \mathrm{C}$ por 24 horas. A MIC foi a menor concentração em que não houve crescimento visível. Paralelamente, 
foi realizado o controle do inóculo bacteriano de todas as amostras por meio de diluições seriadas, seu plaqueamento, incubação e contagem das colônias para confirmar a concentração do inóculo em teste entre $1 \times 10^{5}$ a $1 \times 10^{6} \mathrm{UFC} / \mathrm{ml}$.

A MIC por Etest ${ }^{\circledast}$ foi determinada segundo as instruções do fabricante, inoculando a suspensão bacteriana ajustada em 0,5 de McFarland em ágar Mueller Hinton (M. C. Barth, Porto Alegre, RS), deposição da fita e incubação a $35^{\circ} \mathrm{C}$ por 24 horas. A leitura foi feita no ponto em que a elipse interceptou a tira de Etest.

Dados da MIC obtidos pela automação (MicroScan Walk Away 96, Siemens ${ }^{\circledR}$ ) também foram utilizados. Paralelamente foram usadas cepas ATCC para controle de qualidade: S. aureus ATCC 29213 (mecA positiva) e ATCC 25923 (mecA negativa).

\section{Análise estatística}

Os resultados foram analisados no software Statistical Package for Social Sciences (SPSS) 17.0 e usou-se teste T de Student para amostras independentes.

\section{Resultados}

Os isolados testados apresentaram MICs entre 0,5 e $2 \mu \mathrm{g} / \mathrm{ml}$ (Tabela), resultando em $100 \%$ de sensibilidade à vancomicina segundo critérios do Clinical and Laboratory Standards Institute $(\mathrm{CLSI})^{(2)}$.

Os resultados encontrados pela macrodiluição em caldo e Etest ${ }^{\circledR}$ (Tabela) revelaram concordância de $32,6 \%$ entre os dois métodos, porém é necessário considerar as diferentes concentrações empregadas em cada metodologia. Arredondando a concentração presente somente no Etest ${ }^{\circledR}$ para uma diluição acima, a concordância alcança 39\%.
A maior discordância encontrada foi na concentração de $0,5 \mu \mathrm{g} / \mathrm{ml}$; metade das amostras avaliadas apresentou esse MIC por meio da macrodiluição diante de apenas 2,2\% no emprego de Etest ${ }^{\circledR}$.

Por intermédio da análise estatística, o método comercial $\left(\right.$ Etest $\left.^{\circledR}\right)$ apresentou valores de MIC maiores que de macrodiluição, demonstrando que existe diferença significativa entre as metodologias $(p=0,002)$.

$\mathrm{Na}$ automação (MicroScan Walk Away 96, Siemens ${ }^{\circledR}$ ) todas as amostras apresentaram o valor $\leq 2 \mu \mathrm{g} / \mathrm{ml}$.

\section{Discussão}

No presente estudo não foram detectadas amostras com sensibilidade diminuída à vancomicina, de acordo com os critérios do CLSI (2010). Porém, devido ao uso massivo desse antimicrobiano, é esperado que cepas com perfil de resistência aumentado venham a ser cada vez mais encontradas, justificando seu monitoramento constante por meio de técnicas laboratoriais adequadas.

Os valores de MIC encontrados pelo Etest ${ }^{\circledR}$ foram maiores quando comparados com os da macrodiluição, demonstrando que a técnica tende a superestimar os valores expressos mediante àquela preconizada pelo CLSI. Resultados semelhantes já foram descritos em outros estudos, comparando diluição em caldo e Etest ${ }^{\circledR}$ para determinação de MIC de vancomicina ${ }^{(10,14,18,23)}$.

Uma evidente dificuldade que se encontra ao compararmos testes de diluição em caldo com o Etest $^{\circledR}$ reside na ausência de concentrações totalmente equivalentes em ambos os testes. Ainda assim, um estudo foi realizado utilizando as mesmas concentrações do Etest ${ }^{\circledR}$ na microdiluição, e o resultado encontrado foi semelhante ao dos demais trabalhos, ou seja, o método comercial apresentou MICs mais elevadas ${ }^{(10)}$.

\section{Distribuição de isolados MRSA de acordo com valores de MIC para vancomicina versus metodologia} Tabela utilizada

\begin{tabular}{|c|c|c|c|c|c|c|}
\hline & $\begin{array}{c}0,5 \\
\mu g / m l\end{array}$ & $\begin{array}{c}0,75 \\
\mu \mathrm{g} / \mathrm{ml}^{*}\end{array}$ & $\begin{array}{c}1 \\
\mu g / m l\end{array}$ & $\begin{array}{c}1,5 \\
\mu g /\left.m\right|^{*}\end{array}$ & $\begin{array}{c}2 \\
\mu \mathrm{g} / \mathrm{ml}\end{array}$ & Total \\
\hline \multicolumn{7}{|c|}{$\%(n)$} \\
\hline Macrodiluição & $50(23)$ & N.A. & $45,7(21)$ & N.A. & $4,3(2)$ & $100(46)$ \\
\hline Etest $^{\circledR 1}$ & $2,2(1)$ & $28,3(13)$ & $54,3(25)$ & $13(6)$ & $2,2(1)$ & $100(46)$ \\
\hline Etest $^{\circledR 2}$ & $2,2(1)$ & - & $86,2(38)^{2}$ & - & $15,2(7)^{2}$ & $100(46)$ \\
\hline
\end{tabular}

MRSA: Staphylococcus aureus resistentes à oxacilina; MIC: concentração inibitória mínima; ${ }^{*}$ Concentração presente somente na fita Etest ${ }^{\oplus}$; : MIC Etest lido na fita; ${ }^{2}$ : MIC Etest com arredondamento para uma diluição acima para fins comparativos com a macrodiluição; N.A.: não se aplica. 
Nesse contexto, há um significativo problema na interpretação da sensibilidade à vancomicina por Etest ${ }^{\circledR}$ em cepa que esteja no limite entre sensível $(\leq 2 \mu \mathrm{g} / \mathrm{ml})$ e intermediário $(>2 \mu \mathrm{g} / \mathrm{ml}$ ), pois ela pode ser classificada equivocadamente como não sensível e o paciente receberá tratamento alternativo impróprio. Considerando as evidên-

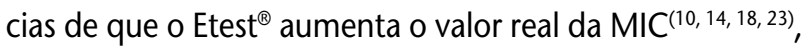
foi inusitado constatar que apenas um isolado MRSA em estudo apresentou MIC $=2 \mu \mathrm{g} / \mathrm{ml}$, ao passo que duas amostras o foram mediante técnica de macrodiluição (padrão). Por outro lado, o Etest ${ }^{\circledR}$ pode ter seu uso encorajado por ser um método de fácil e rápida execução, pouco trabalhoso quando em comparação com a macrodiluição, mas de maior custo. Não se deve omitir este importante aspecto dessa metodologia: a superestimação do valor da MIC. Recomenda-se a confirmação da MIC por meio de técnica de diluição quando o valor encontrado for superior a $1,5 \mu \mathrm{g} / \mathrm{ml}$, a fim de permitir segurança terapêutica. Em paralelo, na escolha do método para rotina, o laboratório deve considerar não somente o preço dos insumos, mas também o custo da mão de obra para executar esses métodos. Nesse âmbito, a macrodiluição pode acabar tornandose mais cara para o laboratório do que o método comercial. Entretanto, ainda existe a possibilidade de uso das placas de microdiluição já disponíveis no mercado nacional.

As cepas VRSA ainda são raras ${ }^{(20)}$, mas o aumento de isolados com heterorresistência ( $h V I S A$ ) e resistência intermediária (VISA) tem sido reportado, inclusive no Brasil ${ }^{(11,13)}$. Um estudo que avaliou as MICs de cepas MRSA diante da vancomicina, de 2001 a 2005, demonstrou que estas vêm aumentando, o que é motivo de preocupação(22). É relevante considerar que todas as VISA/hVISA provêm de pacientes previamente infectados com linhagens MRSA(6).

$O$ incremento da falha terapêutica em infecções causadas por isolados considerados sensíveis (MIC $\leq 2 \mu \mathrm{g} / \mathrm{ml}$ ) ocorre, principalmente, devido a cepas hVISA, ou seja, uma subpopulação com sensibilidade diminuída à vancomicina. Dessa forma, um resultado sensível pode não ser suficiente para o médico determinar o tratamento adequado ${ }^{(18,19,21)}$.

No presente estudo, especula-se que entre as cepas com MIC $=2 \mu \mathrm{g} / \mathrm{ml}$ possam existir linhagens hVISA, cuja detecção laboratorial é muito difícil(7). Quando a MIC de vancomicina aumenta de 0,5 para $2 \mu \mathrm{g} / \mathrm{ml}$, eleva também o grau de falha terapêutica, mesmo que as cepas sigam categorizadas como sensíveis ${ }^{(19)}$. Um trabalho que correlacionou MICs desse fármaco com eficácia clínica mostrou que nos isolados com MIC $\leq 1 \mu \mathrm{g} / \mathrm{ml}$ o êxito do tratamento chega a $48 \%(10 / 21)$, enquanto naquelas com valores $\geq 1 \mu \mathrm{g} / \mathrm{ml}$ o sucesso terapêutico não excede $17 \%(7 / 42)^{(12)}$.

A provável causa para predição de pobre resposta clínica da vancomicina em isolados com $\mathrm{MIC}=2 \mu \mathrm{g} / \mathrm{ml}$ é atribuída a características de farmacocinética/farmacodinâmica (PK/PD). Nesse contexto, o não alcance da relação de área sob a curva de concentração da droga no soro (AUC)/MIC $\geq 400$ compromete o êxito clínico ${ }^{(15)}$. Esse panorama parece ainda menos favorável, uma vez que existem evidências de que cepas com MIC até $1 \mu \mathrm{g} / \mathrm{ml}$ também possam ser hVISA(24).

Diante disso, as MICs obtidas por diluição (CLSI) no presente trabalho evidenciam uma parcela importante de amostras (50\%) nas quais pode ter ocorrido falha terapêutica no uso de vancomicina: $45,7 \%$ (21 isolados) com MIC = $1 \mu \mathrm{g} / \mathrm{ml} \mathrm{e} \mathrm{4,3 \%} \mathrm{(dois} \mathrm{isolados)} \mathrm{com} \mathrm{MIC}=2 \mu \mathrm{g} / \mathrm{ml}$ (Tabela). Devido ao caráter transversal do estudo, o acompanhamento do desfecho dos pacientes com infecção por MRSA em tratamento não foi realizado, mas indiscutivelmente seria um dado relevante a ser considerado.

Os dados oriundos da automação não puderam ser comparados com as demais metodologias, pois o único valor expresso para os isolados sensíveis correspondeu a $\leq$ $2 \mu \mathrm{g} / \mathrm{ml}$. Nesse aspecto, a automação é desfavorável, pois impede o conhecimento da verdadeira MIC do isolado MRSA e, consequentemente, a previsão de sucesso/falha terapêutica no emprego da vancomicina.

Do ponto de vista clínico, o manejo da terapia com vancomicina em infecções por MRSA deve considerar a PK e a PD do referido fármaco, respeitando os níveis considerados seguros para que não haja toxicidade ${ }^{(15)}$.

\section{Conclusão}

A macrodiluição em caldo recomendada pelo CLSI pode ser uma técnica não reprodutível na rotina laboratorial, embora outros métodos não ofereçam a precisão desejada. Porém, a diluição em caldo pode ser realizada em placas de microdiluição já encontradas no mercado nacional.

O Etest ${ }^{\circledR}$ pode ser adotado como alternativa, mas é conveniente que as cepas que apresentarem valores de $\mathrm{MIC}=2 \mu \mathrm{g} / \mathrm{ml}$ possam ser confirmadas por metodologia de diluição em caldo.

A automação exige testes adicionais para reportar o resultado real da MIC, visando a um melhor tratamento, visto que não discrimina o verdadeiro valor da MIC em $0,5,1$ ou $2 \mu \mathrm{g} / \mathrm{ml}$. 


\section{Referências}

1. CENTERS for Disease Control and Prevention (CDC). Disponível em: <http://www.cdc.gov/mrsa/diagnosis/ index.html>. Acesso em: 26 nov. 2010.

2. CLINICAL and Laboratory Standards Institute (CLSI). Performance standards for antimicrobial suscetibility testing: nineteenth informational suplement. CLSI document M100-S19. Wayne, Pennsylvania, USA, 2009.

3. CUEVAS, O. et al. Evolution of the antimicrobial resistance of Staphylococcus spp. in Spain: five nationwide prevalence studies, 1986 to 2002. Antimicrob Agents Chemother, v. 48, n. 11, p. 4240-5, 2004.

4. DAGATA, E. M. C. et al. Modeling the invasion of communityacquired methicillin-resistant Staphylococcus aureus into hospitals. Clin Infect Dis, v. 48, n. 3 , p. 274-84, 2009.

5. DAUNER, D. G.; ROBERT, N. E.; TAKETA, D. C. Ceftobiprole: a novel broad-spectrum cephalosporin with activity against methicillin-resistant Staphylococcus aureus. Am J Health Syst Pharm, v. 67, n 12. p. 983-93, 2010.

6. FRIDKIN, S. Vancomycin-intermediate and -resistant Staphylococcus aureus: what the infectious disease specialist needs to know. Clin Infect Dis, v. 32, n. 1, p. 108-15, 2001

7. HOWDEN, B. P. et al. Reduced vancomycin susceptibility in Staphylococcus aureus, including vancomycinintermediate and heterogeneous vancomycinintermediate strains: resistence mechanisms, laboratory detection, and clinical implications. Clin Microbiol Rev, v. 23, n. 1, p. 99-139, 2010.

8. LIVERMORE, D. M.; MUSHTAQ, S.; WARNER, M. Activity of the anti-MRSA carbapenem razupenem (PTZ601) against Enterobacteriaceae with defined resistant mechanisms. J Antimicrob Chemother, v. 64, n. 2, p. 330-5, 2009.

9. LUCET, J. C. et al. Prevalence and risk factors for carriage of methicillin-resistant Staphylococcus aureus at admission to the intensive care unit: results of a multicenter study. Arch Intern Med, v. 163, n. 2, p. 181-8, 2003.

10. MASON, E. O. et al. Vancomycin MICs for Staphylococcus aureus vary by detection method and have subtly increased in a pediatric population since 2005. J Clin Microbiol, v. 47, n. 6, p. 1628-30, 2009.

11. MELO, G. B. et al. Staphylococcus aureus e estafilococos coagulase negativos resistentes à vancomicina em um Hospital Universitário Brasileiro. Rev Ciênc Farm Básica Apl, v. 30, n. 1, p. 45-50, 2009.

12. MOISE-BRODER, P. A. et al. Accessory gene regulator group ii polymorphism in methicillin-resistant Staphylococcus aureus is predictive of failure of vancomycin therapy. Clin Infec Dis, v. 38, n. 12, p. 1700-5, 2004.

13. OLIVEIRA, G. A. et al. Avaliação da tolerância à vancomicina em 395 cepas hospitalares de Staphylococcus aureus resistentes à oxacilina. J Bras Patol, v. 37, n. 4, p. 239-46, 2001.

14. PRAKASH, V. et al. Vancomycin MICs for methicillinresistant Staphylococcus aureus isolates differ based upon the susceptibility test method used. Antimicrob Agents Chemother, v. 52, n. 12, p. 4528, 2008.
15. RIBAK, M. Therapeutic monitoring of vancomycin in adult patients: a consensus review of the American Society of Health-System Pharmacists, the Infectious Diseases Society of America, and the Society of Infectious Diseases Pharmacists. Am J Health Syst Pharm, v. 66, p. 82-98, 2009.

16. RIBEIRO, A. et al. First report of infection with communityacquired methicillin-resistant Staphylococcus aureus in South America. J Clin Microbiol, v. 43, n. 4, p. 1985-8, 2005.

17. ROSSI, F.; ANDREZZI, D. Resistência bacteriana: interpretando o antibiograma. São Paulo: Atheneu, 2006.

18. SADER, H. S.; RHOMBERG, P. R.; JONES, R. N. NineHospital Study comparing broth microdilution and Etest method results for vancomycin and daptomycin against methicillin-resistant Staphylococcus aureus. Antimicrob Agent Chemoter, v. 53, n. 7, p. 3162-5, 2009.

19. SAKOULAS, G. Relationship of MIC and bactericidal activity to efficacy of vancomycin for treatment of methicillinresistant Staphylococcus aureus bacteremia. J Clin Microbiol, v. 42, n. 6, p. 2398-402, 2004.

20. SIEVERT, D. M. et al. Vancomycin-resistant Staphylococcus aureus in the United States, 2002-2006. Clin Infect Dis, v. 46, n. 5 , p. $668-74,2008$

21. SORIANO, A. et al. Influence of vancomycin minimum inhibitory concentration on the treatment of methicillinresistant Staphylococcus aureus bacteremia. Clin Infect Dis, v. 46, n. 2, p. 193-200, 2008.

22. STEINKRAUS, G. E.; WHITE, R.; FRIEDRICH, L. Vancomycin MIC creep in non-vancomycinintermediate Staphylococcus aureus (VISA), vancomycinsusceptible clinical methicillin-resistant $S$. aureus (MRSA) blood isolates from 2001-05. J Antimicrob Chemother, v. 60, p. 788-94, 2007. Disponível em: <http://www.triusrx. com/pdfs/Nancomycin_MIC_creep_in_non-vancomycinintermediate.pdf>. Acesso em: 23 mar. 2010.

23. SWENSON, J. M. et al. Accuracy of commercial and reference susceptibility testing methods for detecting vancomycin-intermediate Staphylococcus aureus. J Clin Microbiol, v. 47, n. 7, p. 1628-30, 2009.

24. TENOVER, F. C.; MOELLERING JUNIOR, R. C. The rationale for revising the clinical and laboratory standards institute vancomycin minimal inhibitory concentration interpretative criteria for Staphylococcus aureus. Clin Infect Dis, v. 44, p. 1208-15, 2007.

25. TIWARI, H. C.; SEN, M. R. Emergence of vancomicyn resistance Staphylococcus aureus (VRSA) from a tertiary care hospital from northern part of India. BMC Infect Dis, v. 6, n. 156, 2006. Disponível em: <http:// www. biomedcentral.com/1471-2334/6/156>. Acesso em: 14 set. 2009
Endereço para correspondência

Caroline Manfredin Rua Caribaldi, 776 CEP: $95700-000$ - Bento Conçalves-RS e-mail: carolmanfredini@gmail.com 